Department of Economics

and

Center for Research on Economic and Social Theory

Working Paper Series

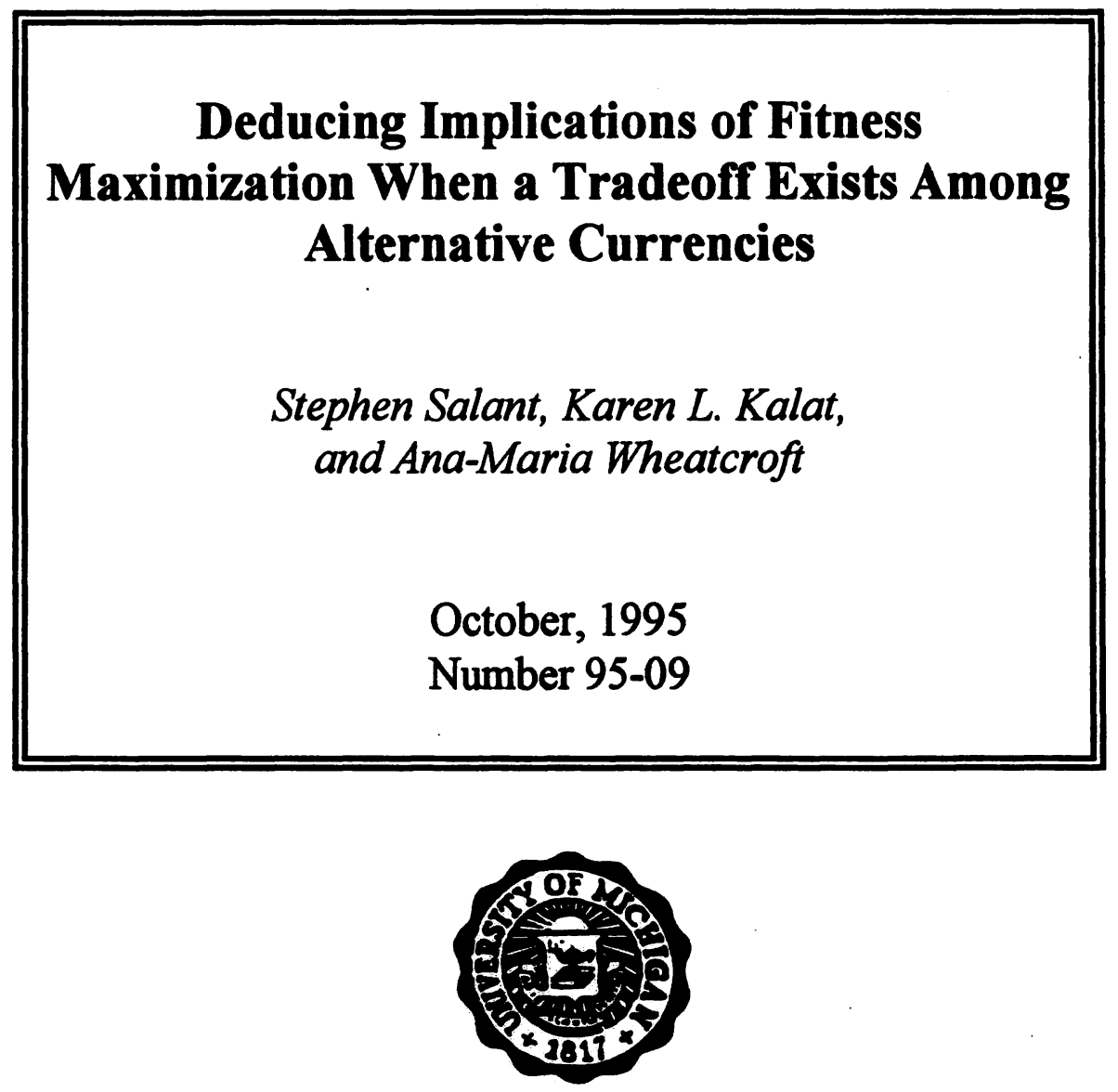

DEPARTMENT OF ECONOMICS

University of Michigan

Ann Arbor, MI 48109-1220 



\section{Deducing Implications of Fitness Maximization When a Tradeoff Exists Among Alternative Currencies}

Stephen W. Salant---Department of Economics, University of Michigan Karen L. Kalat---Division of Biostatistics, School of Public Health, University of

California, Berkeley
Ana-Maria Wheatcroft---Morgan Stanley \& Co., Inc., New York City, NY
While the theory of natural selection posits that those behaviors maximizing reproductive success ("fitness") tend to survive, behavioral ecologists frequently explain observed behaviors as maximizing some "currency" on which fitness depends. A weakness of the approach is that reproductive success often depends on more than one currency and behaviors which augment one currency may reduce another. We explain how to deduce from the hypothesis of fitness maximization testable predictions. We expound the approach entirely in terms of two biological examples---a preliminary example involving flower replacement perennial and a more elaborate on involving over-winter hoarding by a female mammals.

JEL Classification: A12, B1, D61, Q20

Keywords: currency, economic efficiency, fitness, foraging, hoarding, optimal control, optimization 
In the last two decades, behavioral ecologists have developed a variety of models to explain foraging and other "behaviors" of plants and animals from an optimization perspective. Although the theory of natural selection posits that behaviors maximizing reproductive success or fitness tend to survive, most modellers in fact modify this hypothesis and assume instead that some other proxy or "currency" is maximized. For example, in the case of optimal foraging theory the long-term rate of energy intake is almost invariably used as a proxy for fitness. In their valuable survey of this literature, however, Stephens and Krebs (1986) point out that reproductive success often depends on more than this single currency. Since behaviors which promote energy gain may at the same time impede other factors contributing to reproductive success, tradeoffs inevitably exist. As Stephens and Krebs put it: “The best feeding site may be the most dangerous, the worst place to find a mate, or the least suitable for building a nest." Our purpose in this paper is to describe a methodology for characterizing optimal behavior when reproductive success depends on multiple currencies over which tradeoffs exist. As we show, this approach is especially useful when comparatively little is known about the form of the fitness function. We illustrate the approach in subsequent sections by means of two examples.

In our first example, we consider a perennial which produces flowers during part of the year and then lies dormant until the next flowering season. Energy is required to generate each flower; moreover, energy is needed to maintain it. Each flower produces seeds. The longer a given flower remains on the plant, the more seeds are produced but the production occurs at a decreasing rate. In principle, the plant might generate any number of flowers (including zero) over the course of the season. Moreover, it might replace each flower after the same length of time or it might replace some after different lengths of time.

These behaviors are hypothesized to maximize fitness. Fitness depends on two currencies. It presumably strictly increases with the number of seeds produced during the season; but, for any given number of seeds produced, fitness may also depend on the energy reserves of the plant at the end of the flowering season. Since increasing seed production ultimately means that the plant has less energy reserves at the season's end, a tradeoff inevitably exists between these two determinants of fitness. Unfortunately, relatively little is known about the "fitness function" compared to the detailed knowledge of the energy costs of producing and maintaining flowers and the details of seed production. What if anything can be said in these circumstances about the implications of the hypothesis of fitness maximization?

In our second example, we consider a female mammal such as a squirrel. Throughout the year, the squirrel finds food and either consumes or stores it. A fraction of the stored food decays, is stolen or cannot be re-located. However, the remainder may be either retrieved and consumed by the female or may be saved for her next offspring. The activities of finding, storing and retrieving food each period require energy. Moreover, finding a given amount of food after winter arrives requires more energy than before the onset of winter. The weight gain of the fernale in a given period depends on her food consumption and energy expenditure then. In principle, the female squirrel might exhibit any of a variety of behavioral patterns over the course of the year for finding, storing, retrieving, and consuming food.

How the squirrel behaves is hypothesized to maximize fitness. Fitness depends on two currencies. It presumably strictly increases with the food stored for the offspring when the next litter arrives; but, for any given amount of food in storage, fitness may also depend on the female's body weight then. Since increasing the food in storage for the next litter ultimately means that the female has less body weight when the litter arrives, a tradeoff inevitably exists between these two determinants of fitness. Unfortunately, little is known about the "fitness function" compared to the detailed knowledge of the energy costs of finding, storing, and retrieving food, and the weight gain and loss associated respectively with consumption and energy expenditure. What if anything can be said in these circumstances about the implications of the hypothesis of fitness maximization?

Quite a lot. In the case of the perennial, for example, we can conclude as a qualitative matter that each flower will be maintained for the same length of time. In the case of the female squirrel, predictions will depend in part on the pattern of food availability before and after the onset of winter. In the stylized case where 
food is uniformly available prior to the onset of winter and uniformly scarce thereafter, for example, the female's storage and overall activity will increase as winter approaches. After the onset of winter, retrieval replaces storage, foraging may be suspended entirely (although this stage will be skipped under circumstances we delineate in the Appendix), and overall activity drops. As time passes, retrieval declines and-possibly after decreasing for a while-overall activity begins to increase until the next litter arrives. Finally, females with larger body weights are predicted to have higher reproductive success.

In addition to these qualitative deductions from the hypothesis of fitness maximization, quantitative deductions are possible if some additional information is supplied about the observed behavior. In the case of the perennial, for example, we could deduce both the number of flowers the plant would produce and also the length of time each flower would be maintained if we observed the energy reserves of the plant at the end of the flowering season. In the case of the squirrel, we could predict the amount of consumption, storage, retrieval, and foraging the squirrel would undertake throughout the year if we observed the weight gain of the squirrel between the beginning and end of the year. Our formulation of this second example permits both qualitative and quantitative analysis even when food availability exhibits systematic seasonal variation prior to the onset of winter.

How can such qualitative and quantitative deductions be made from the hypothesis of fitness maximization when so little is known about the fitness function itself? The remainder of this paper explains the approach in the context of these two examples. Our methodology lies at the core of modern economics. There, tradeoffs are represented by the celebrated "transformation curve" and the maximand is referred to as the "social welfare function." Although the form of this function is rarely specified, economists nonetheless deduce many propositions (referred to as "efficiency conditions") about the underlying behaviors which maximize it. Our purpose here is to clarify the logical steps leading to such deductions by applying the approach to our two biological examples. Readers interested in seeing the method we describe applied to economic problems can consult the first few pages of the prominent graduate text in microeconomics by Layard and Walters (1978) or, for a more detailed treatment concentrating on dynamic problems like those we discuss, sections on "intertemporal efficiency conditions" in Dorfman, Samuelson, and Solow (1987).

\section{Tradeoff between Seed Production and Energy Re- serves}

Schoen and Ashman (in press) have modelled the evolution of optimal flower longevity in cases where the resources available for flower production in a single year can be allocated either to maintenance of existing flowers or to the construction of additional flowers. Here we consider the case of a perennial plant. In such a plant, resources allocated to flower production and maintenance in one year influence those available in future years.

During the flowering season, a perennial plant creates $n$ flowers. Let $a_{i}(i=$ $1, \ldots, n)$ denote the age of flower $i$ in days when it is replaced. Let $s\left(a_{i}\right)$ denote the cumulative number of seeds produced (either directly or, if its pollen is spread to other plants, indirectly) by a flower replaced at age $a_{i}$. We assume that the longer a flower is maintained, the more seeds are produced but at a decreasing rate. That is, $s(\cdot)$ is strictly increasing and strictly concave; for simplicity, assume also that it is differentiable. During the season the perennial will generate $\sum_{i=1}^{n} s\left(a_{i}\right)$ seeds.

Let $\tilde{E}$ denote the energy reserves the perennial would have at the end of the season if it created no flowers. Assume that each flower costs $c$ units of energy to create and $m$ units of energy per day (net) to maintain. Then the energy reserves of the plant at the season's end, denoted $\hat{E} \geq 0$, will satisfy:

$$
\hat{E}=\bar{E}-n c-m \sum_{i=1}^{n} a_{i},
$$

where $n$ is a nonnegative integer and $a_{i} \geq 0$ for $i=1, \ldots, n$.

Each "behavior" (number of flowers, $n$, and their respective replacement times, $\left.\left\{a_{i}\right\}\right)$ yields an "outcomen : a quantity of seeds produced $(S)$ and an energy reserve at the season's end $(\hat{E})$. The set of possible outcomes lies either $(a)$ on the boundary 
of Figure 1 or (b) in the interior area between this boundary and the axes. Assuming that the perennial's reproductive success is a strictly increasing function of the number of seeds produced during the season and may depend on end-of-season energy reserves as well, fitness maximization will entail behaviors which lie on-rather than-inside the boundary of Figure 1. For, suppose the contrary. Suppose a point inside the boundary maximized fitness. Then, alternative behaviors exist which would yield an outcome with the same end-of-season energy reserves but greater production of seeds. But such behaviors would entail greater reproductive success. Hence, the supposition must be false.

Consider any point on the boundary in Figure 1 with vertical component $S$ and borizontal component $\hat{E}$. This outcome and the behaviors that underly it can be defined analytically in two alternative ways: by maximizing the horizontal distance given a vertical height $S$ or by maximizing the vertical height given horizontal distance $\dot{E}$. To illustrate, we formulate the latter problem:

$\max _{n,\left\{a_{i}\right\}} \sum_{i=1}^{n} s\left(a_{i}\right)$

subject to $a_{i} \geq 0, n$ an integer

$$
\hat{E}=\bar{E}-n c-m \sum_{i=1}^{n} a_{i} .
$$

Notice that $P 1$ itself does not involve the fitness function. Nonetheless, any behavior maximizing the fitness function must solve this underlying subproblem. Denote the maximized value as $S$.

It turns out that for each $\hat{E}$ the behavior solving the resulting subproblem has a common qualitative feature: each flower is replaced after an equal interval of time. For, suppose it were optimal to create $n^{p}$ flowers and maintain flower $i$ longer than flower $j\left(a_{i}^{p}>a_{j}^{p}\right)$. Denote the resulting outcome as $\sum_{i=1}^{n} s\left(a_{i}^{p}\right), \hat{E}^{p}$. Then it is straightforward to display an alternative behavior which would result in the same end-of-season energy reserves but larger production of seeds, and hence greater reproductive success. Suppose the longer replacement time was shortened and the shorter replacement time was lengthened by the same amount. If no other changes occurred, the end-of-season energy reserves would be unaffected since the same number of flow- ers would be created and the sum of their maintenance times would be unchanged. But strict concavity of $s(\cdot)$ insures that, for sufficiently small perturbations, a larger number of seeds will be produced. In particular, since the sum of the replacement times of flowers $i$ and $j$ is unchanged, $d a_{j} / d a_{i}=-1$. Since $a_{i}^{p}>a_{j}^{p}$, strict concavity of $s(\cdot)$ implies that $s^{\prime}\left(a_{i}^{p}\right)<s^{\prime}\left(a_{j}^{p}\right)$. The total number of seeds produced by the plant will then change at the rate: $d\left[\sum_{i=1}^{n} s\left(a_{i}^{p}\right)\right] / d a_{i}=s^{\prime}\left(a_{i}^{p}\right)-s^{\prime}\left(a_{j}^{p}\right)<0$. Hence, the proposed perturbation would increase seed production. It follows that each outcome on the boundary of Figure 1 must be generated by behaviors where flowers are replaced after equal lengths of time. This implication of the hypothesis of fitness maximization has been deduced without a detailed specification of the fitness function.

It is important to recognize the generality of this formulation. While the reproductive success of the perennial depends on the number of seeds it produces during the current season, its overall reproductive success also depends on its own future seed production as well as the future seed production of each of its descendants ... Redefine $\bar{E}, n$, and $\left\{a_{i}\right\}$ so that they pertain either to the original perennial or to any specified successor alive in any specified future season. Then, if overall reproductive success is maximized, the designated plant-for whatever energy reserve it starts the designated season-presumably could not during that flowering season both produce more seeds and nonetheless end the season with more energy reserves by maintaining a different number of flowers or replacing them at different time intervals. Hence its "behaviors" also solve P1. For this reason, the problem formalized above is fundamental.

There is one other qualitative implication of fitness maximization which can be deduced. We introduce it here although its relevance will become clearer in the animal hoarding example of the next section. Suppose there are regions in which fitness is nonincreasing in end-of-season energy reserves. We can predict that fitness will never be maximized in such a region (except in circumstances which can be disregarded, where end-of-season energy reserves are zero). That is, increases in end-of-season energy reserves must strictly increase fitness in the neighborhood of an optimum.

To establish this, first note that the boundary of Figure 1 slopes dowawards. For, if end-of season energy reserves were reduced, seed production could always be 
increased by using the energy saved to maintain the same number of flowers for longer. Recall that the energy-seed combination where fitness is maximized lies somewhere on this downward-sloping boundary. Now suppose in the neighborhood of the point where fitness is maximized that an increase in end-of-season energy reserves would result in unchanged or reduced fitness. Then alternative behaviors exist which would result in more seeds and less energy reserves at the end of the season. But these alternative behaviors would, by hypothesis, enjoy greater reproductive success. The larger number of seeds would strictly increase fitness while the reduced energy reserves would, by hypothesis, not diminish fitness. We conclude that whenever fitness is maximized a local increase in end-of-season energy reserves must strictly increase reproductive success.

While we can deduce from the hypothesis of fitness maximization these qualitative conclusions, we cannot quantify them without additional information. For example, we can predict that all flowers will last equal lengths of time but we cannot determine the magnitude of the common length nor can we determine the number of flowers that will be produced. To make such quantitative predictions, additional information is necessary.

Suppose we observed as additional information the particular energy reserve of the perennial at the season's end (denoted $\hat{E}^{*}$ ). Given this additional information, we could also deduce quantitative implications of the maximization hypothesis. For, if fitness was maximized, the perennial's behavior must have achieved the point on the boundary of Figure 1 with horizontal component $\hat{E}^{*}$. Since a unique set of behaviors can be shown to underly virtually any point on the boundary of Figure 1, we could deduce quantitative predictions about the number of flowers the plant will produce, the replacement times of each flower, and the number of seeds produced. These quantitative predictions require no additional information about the fitness function.

\section{Tradeoff between Female's Body Weight and Ex- ternal Storage}

In the prior example, we identified as "behaviors" to be predicted the number of flowers $(n)$ and their respective durations $\left(\left\{a_{i}\right\}\right)$, and we required that those behaviors be nonnegative and not overexhaust the energy reserves of the plant. Each collection of feasible behaviors yields an outcome-a combination of seed production and endof-season energy reserves-which by hypothesis affects fitness. We represented the set of such outcomes in Figure 1. If the fitness function is strictly increasing in at least one of these "currencies," then fitness maximization requires that the behaviors result in a point on the boundary. Behaviors resulting in any interior point never maximize fitness. Each boundary point and the behaviors underlying it can be characterized analytically by maximizing the vertical distance above each point on the horizontal axis of Figure 1 (or, alternatively, by maximizing the horizontal distance to the right of each point on the vertical axis).

In the animal hoarding example, we proceed in exactly the same way. How the animal conducts the following five activities over time affects fitness: the amount of food harvested, newly stored, retrieved and consumed as well as the energy expended on these food-related activities. We refer to these as behavioral variables:

Behavioral Variables

$$
\begin{aligned}
& H_{t}=\text { units of food harvested (foraged) in period } t ; \\
& N_{t}=\text { units of food newly-stored in period } t ; \\
& R_{t}=\text { units of food retrieved from storage in period } t ; \\
& C_{t}=\text { units of food consumed in period } t ; \\
& A_{t}=\text { energy expended in period } t \text { in food-related activities. }
\end{aligned}
$$

Since these behavioral variables are interrelated and none can be negative, we impose the following feasibility restrictions:

$$
C_{t}=H_{t}+R_{t}-N_{t}, \text { for } t=1, \ldots, T
$$




$$
\begin{gathered}
A_{t}=\alpha_{h(t)} H_{t}+\alpha_{r}(t) R_{t}+\alpha_{n}(t) N_{t}, \text { for } t=1, \ldots, T \\
A_{t}, N_{t}, C_{t}, H_{t}, R_{t} \geq 0,
\end{gathered}
$$

where $T$ is the period when the first post-winter litter arrives.

The first of these equations requires that the two uses of food (consumption and new storage) equal the two sources of food (food retrieved from storage and food just foraged). The second equation indicates that the total energy expended in period $t$ on food-related activities is a linear function of the food stored, retrieved, or currently harvested then. Since it requires more energy to pursue these activities at different times of the year, the coefficients of the linear function may vary over time.

Next, we clarify how feasible behaviors result in outcomes on which fitness depends. We assume that initially the female's body weight and the stockpile size are given. Denote them by $\bar{W}$ and $\bar{S}$, respectively. By choosing five feasible behaviors in each period, the animal alters her body weight and stockpile size over time. We define the state variables $S_{t}$ and $W_{t}$ as follows:

\section{State Variables}

$S_{t}=$ food in storage at the beginning of period $t ;$

$W_{t}=$ weight of mother at the beginning of period $t$.

Fitness is assumed to depend both on the female's body weight when her first postwinter litter arrives $\left(W_{T+1}\right)$ and on food in storage then $\left(S_{T+1}\right)$. We refer to these variables as "currencies." The size of the stockpile at $t+1$ equals a fixed proportion $(\theta)$ of its size at $t$ after adjustment for deposits or withdrawals in that period. The complementary proportion $(1-\theta)$ is assumed to be irretrievable afterwards; it either rots, is stolen or cannot be relocated. The female's body weight at $t+1$ equals the sum of her body weight at the beginning of period $t$ and her weight gain $\left(G\left(C_{t}, A_{t}, W_{t}, t\right)\right)$ during that period. Her weight gain in turn depends on her consumption, energy expenditure, and mass as well as on the temperature at that time of year. When the gain function is negative, we interpret it as a loss in weight:

$$
S_{t+1} \leq\left(S_{t}+N_{t}-R_{t}\right) \theta, \text { for } t=1, \ldots, T
$$

$$
\begin{gathered}
W_{t+1}=W_{t}+ \\
G\left(C_{t}, A_{t}, W_{t}, t\right), \text { for } t=1, \ldots, T \\
S_{1} \leq \bar{S}, W_{1}=\bar{W}, \\
\text { and } S_{t}, W_{t} \geq 0 .
\end{gathered}
$$

Any feasible $5 T$ behaviors (five behaviors in each of $T$ periods) generates a pair of outcomes on or inside the boundary in Figure 2. Many behaviors result in outcomes inside the boundary. If the fitness function is strictly increasing in at least one of these currencies, however, then fitness maximization requires that the behaviors result in a point on the boundary. Behaviors resulting in an interior point never maximize fitness.

To determine if a set of behaviors results in a boundary point, no information about the fitness function is required. The information required is summarized below: 


\section{Inputs to Model}

\section{$\bar{S}=$ initial stock of food in storage;}

$\bar{W}=$ initial body weight of mother;

$\alpha_{h(t)}=$ energy required to forage one unit of food in period $t ;$

$\alpha_{r(t)}=$ energy required to retrieve one unit of food from storage in period $t ;$

$\alpha_{n(t)}=$ energy required to store one unit of food in period $t$;

$\theta=$ the fraction of food retrievable after being stored for one period;

$T=$ the period when the first offspring after winter are born;

$G\left(C_{t}, A_{t}, W_{t}, t\right)=$ the weight gain (or loss, if negative) of the mother in period $t$.

We analyze the problem by maximizing the vertical distance above an arbitrary point $(\hat{S}>0)$ on the horizontal axis of Figure 2:

P2

$$
\text { Maximize } W_{T+1}=\bar{W}+\sum_{t=1}^{T} G\left(C_{t}, A_{t}, W_{t}, t\right)
$$

subject to $S_{t+1} \leq\left(S_{t}+N_{t}-R_{t}\right) \theta$, for $t=1, \ldots, T$

$W_{t+1}=W_{t}+G\left(C_{t}, A_{t}, W_{t}, t\right)$, for $t=1, \ldots, T$

$C_{t}=H_{t}+R_{t}-N_{t}$, fort $=1, \ldots, T$

$A_{t}=\alpha_{n(t)} H_{t}+\alpha_{r(t)} R_{t}+\alpha_{n(t)} N_{t}$, for $t=1, \ldots, T$

$S_{1} \leq \bar{S}, W_{1}=\bar{W}$,

$S_{T+1} \geq \hat{S}$

and $A_{t}, N_{t}, C_{t}, H_{t}, R_{t}, S_{t} \geq 0$.

It is important to recognize the generality of this formulation. While members of the litter born at $T$ clearly benefit from food in storage then and from the mother's body weight, the subsequent offspring of the mother also contribute to ber overall reproductive success as do their offspring... Consider the first litter after the onset of any winter born either to the original mother or to any of her female descendants. Redefine $T$ as the number of periods between that litter and her previous one and reindex the time periods between the two litters from 1 to $T$, with $T$ denoting the period when the later litter arrives. If fitness (in the comprehensive sense) is maxi- mized, it cannot be possible to alter the behavior of any such designated mother in periods 1 through $T$ in such a way that, without affecting her body weight when her litter arrives at $T$, more food remains in storage then. For this reason, the problem formalized above is fundamental.

In our first example, we deduced two types of qualitative predictions from the hypothesis of fitness maximization. First, it was pointed out that behaviors which underlie boundary points in Figure 1 differ qualitatively from behaviors which underlie interior points. In particular, if flowers are replaced after different lengths of time, we proved that the outcome would be an interior point in Figure 1; hence, fitness cannot be maximized. In our animal hoarding example, the description of the qualitative characteristics of paths achieving the boundary of Figure 2 will occupy all of the next section and the Appendix.

Second, it was pointed out in our plant example that if fitness is strictly increasing in one currency but is nonincreasing in another currency in certain regions, then an optimum will never occur in those regions where fitness is nonincreasing. This qualitative proposition also has its counterpart in our animal hoarding example. Suppose fitness is a strictly increasing function of the food in storage when the litter is born. For any given stockpile size, however, suppose that increases in the mother's body weight will strictly increase her fitness if her weight then is below some threshold but will reduce her fitness if her weight then exceeds this threshold (due to obesity). Then, from the hypothesis of fitness maximization we can deduce the prediction that in the neighborhood of the body weight achieved by the animal at the time of her first post-winter litter, a local increase in weight would strictly increase reproductive success. The argument is the same as before. Since fitness is strictly increasing in one currency, fitness maximization must result in a point on the boundary of Figure 2. Since this boundary is downward-sloping, an optimum cannot occur (with $W_{T+1}>0$ ) in a region where fitness is nonincreasing in body weight since slightly different behaviors would then exist which would result in more food in storage and less body weight and hence would result in increased fitness.

Finally, it was pointed out that even quantitative predictions can be deduced with- 
out knowing more about the form of the fitness function if additional information is observed. In our animal hoarding example, an observation of either the animal's weight gain or its stockpile when the litter is born would permit us to predict quantitatively all $5 \mathrm{~T}$ behavioral variables! Recall the argument. Under the hypothesis of fitness maximization, some point on the boundary of Figure 2 will be achieved. Since the additional information specifies one coordinate of this point and there are assumed to exist only two currencies, the second coordinate can easily be determined. The $5 \mathrm{~T}$ behaviors underlying this boundary point can then be predicted quantitatively because the concave constrained optimization problem has a unique solution.

\section{Further Qualitative Analysis of the Animal Hoard- ing Example}

\section{Simplifying Assumptions}

To predict behavior using the foregoing methodology, one does not need to know the form of the fitness function. One does need to know, however, the weight-gain consequences of food intake on the one hand and energy expenditure $\left(G\left(C_{t}, A_{t}, W_{t}, t\right)\right)$ on the other, as well as the energy cost of the alternative food-related activities $\left(\alpha_{h(t)}, \alpha_{n(t)}, \alpha_{r(t)}\right)$. The assumptions we make below facilitate our analysis and seem to us a reasonable starting point.

\section{- Weight-gain}

Abstracting from the dependence of weight gain in period $t$ on the animal's own body weight and on the outside temperature (which varies with $t$ ), we write weight gain as a separable function of the first two variables: $G\left(C_{t}, A_{t}, W_{t}, t\right)=$ $U\left(C_{t}\right)-F\left(A_{t}\right)$. Furthermore, we assume the first and second derivatives of $U(\cdot)$ and $F(\cdot)$ exist and are continuous. Finally, we assume the following:

$$
\begin{array}{ll}
\text { B1: } & U^{\prime}(C)>0, U^{\prime \prime}(C)<0 ; \quad F^{\prime}(A)>0, F^{\prime \prime}(A)>0 . \\
\text { B2: } & F^{\prime}(0) \text { is finite and } \lim _{C \rightarrow 0} U^{\prime}(C)=\infty .
\end{array}
$$

The former assumption insures that the optimum is unique while the latter reduces somewhat the number of cases which can arise as will be clarified below.

\section{- Energy costs}

Next, we assume that each of the food-related activities consumes energy:

$$
\text { B3: } \quad \alpha_{r(t)}>0, \alpha_{n(t)}>0, \alpha_{h(t)}>0 .
$$

Denote the last period before the onset of winter as $t_{w}$. Assumptions we will make about the reduced availability of food during the winter will insure that no retrieval occurs in the model until $t_{w}+1$. Retrieval will occur afterwards only if it costs less in terms of energy than foraging for the same amount of food:

$$
\text { B4: } \quad \alpha_{h(t)}>\alpha_{r(t)} \text { for } t=t_{w}+1, \ldots, T .
$$

We assume that the energy required to store (or to retrieve) food is the same over the $T$-period horizon:

$$
\text { B5: } \quad \alpha_{r(t)}=\alpha_{r} \text { and } \alpha_{n(t)}=\alpha_{n} .
$$

As we will see, if all stored food could be retrieved without loss from rot, theft, or misplacement and if, in addition, food were both uniformly available before the onset of winter and uniformly unavailable thereafter, fitness maximization would require that the behavior of the animal in every pre-winter period be identical; and a different but unchanging behavior would be required in each period after the onset of winter. This recurrent behavior is reminiscent of our first example where each flower is replaced after the same length of time no matter when in the season it initially forms.

However, two forces independently create a rich dynamics in the hoarding example. The first, which is hardly surprising, is that the availability of food systematically varies before the onset of winter. For example, squirrels store mast which becomes more available as winter approaches.

The second force, which is more surprising, results from the fact that some stored food is irretrievable: since $\theta<1$, the stockpile can be thought of as a sieve. Even if food were uniformly available before the onset of winter and uniformly unavailable thereafter (until the arrival of the next litter), a complicated pattern of behavior over time would result simply from optimal responses to 
leakage from storage $(\theta \in(0,1))$. We refer to such induced behaviors as "sieve effects. ${ }^{n}$ When we wish to isolate these effects, we will find it useful to invoke the following assumption:

$$
\text { B6: } \quad \alpha_{h(t)}= \begin{cases}\underline{\alpha} & \text { if } t=1,2, \ldots t_{w} \\ \alpha>\underline{\alpha} & \text { otherwise }\end{cases}
$$

Henceforth, each mention of an assumption is set in boldface to facilitate identifying where each assumption is used.

\section{Conditions Defining Each Optimum}

To analyze the constrained maximization problem (P2) posed in the previous section, it is convenient first to substitute out the variable $C_{t}$. Then, forming the Lagrangean we obtain:

$$
\begin{aligned}
L=\sum_{t=1}^{T}\{ & U\left(H_{t}+R_{t}-N_{t}\right)-F\left(A_{t}\right)+\lambda_{t}\left[\theta_{t}\left(S_{t}+N_{t}-R_{t}\right)-S_{t+1}\right] \\
& \left.+\gamma_{t}\left[A_{t}-\left(\alpha_{h(t)} H_{t}+\alpha_{r} R_{t}+\alpha_{n} N_{t}\right)\right]\right\} \\
& +\lambda_{0} \theta\left(\bar{S}-S_{1}\right)+\theta \lambda_{T+1}\left(S_{T+1}-\hat{S}\right)
\end{aligned}
$$

The following Kuhn-Tucker conditions must hold if a program is optimal. For $t=1,2, \ldots, T$,

1. $H_{t} \geq 0 ; \frac{\partial L}{\partial H_{t}}=U^{\prime}\left(H_{t}+R_{t}-N_{t}\right)-\gamma_{t} \alpha_{h(t)} \leq 0$; with complementary slackness;

2. $R_{t} \geq 0 ; \frac{\partial L}{\partial R_{t}}=U^{\prime}\left(H_{t}+R_{t}-N_{t}\right)-\lambda_{t} \theta-\gamma_{t} \alpha_{r} \leq 0$; with complementary slackness;

3. $N_{t} \geq 0 ; \frac{\partial L}{\partial N_{t}}=-U^{\prime}\left(H_{t}+R_{t}-N_{t}\right)+\lambda_{t} \theta-\gamma_{t} \alpha_{n} \leq 0$; with complementary slackness;

4. $S_{t} \geq 0 ; \frac{\partial L}{\partial S_{t}}=\lambda_{t} \theta-\lambda_{t-1} \leq 0$; with complementary slackness;

5. $S_{T+1} \geq 0 ; \frac{\partial L}{\partial S_{T+1}}=\theta \lambda_{T+1}-\lambda_{T} \leq 0$; with complementary slackness;

6. $\lambda_{t} \geq 0 ; \frac{\partial L}{\partial \lambda_{t}}=\theta\left(S_{t}+N_{t}-R_{t}\right)-S_{t+1} \geq 0$; with complementary slackness;
7. $\lambda_{0} \geq 0 ; \frac{\partial L}{\partial \lambda_{0}}=\theta\left(\bar{S}-S_{1}\right) \geq 0$; with complementary slackness;

8. $\quad \frac{\partial L}{\partial \gamma_{t}}=A_{t}-\left(\alpha_{h(t)} H_{t}+\alpha_{r} R_{t}+\alpha_{n} N_{t}\right)=0$

9. $\lambda_{T+1} \geq 0 ; \frac{\partial L}{\partial \lambda_{T+1}}=\theta\left(S_{T+1}-\hat{S}\right) \geq 0$; with complementary slackness; and

10. $A_{t} \geq 0, \frac{\partial L}{\partial A_{t}}=-F^{\prime}\left(A_{t}\right)+\gamma_{t} \leq 0$; with complementary slackness.

As explained in Baumol (1977) "complementary slackness" is a shorthand term meaning that at least one of the two weak inequalities in the list must equal zero. Thus, for example, Condition 1 indicates not merely that $H_{t} \geq 0$ and $\frac{\partial L}{\partial H_{t}} \leq 0$ but that whenever $H_{t}>0, \frac{\partial L}{\partial H_{t}}=0$ and whenever $\frac{\partial L}{\partial H_{t}}<0, H_{t}=0$. The Kuhn-Tucker conditions are a systematic if somewhat mechanical way of taking into account all the equality and inequality constraints in an optimization problem. A more intuitive and equivalent way of proceeding would be to show that whenever a feasible program violates one or more Kuhn-Tucker conditions, there exists a different feasible program yielding a larger bodyweight but the same size stockpile. While the use of the KuhnTucker conditions saves journal space, some readers may prefer a derivation of our results which does not rely on these conditions; they should consult Section 3 of our working paper (Salant et al., 1993). Henceforth, we use the term "Condition" (followed by its number) when referring to a complementary slackness condition in its entirety and "Equation" (followed by its number) when referring to either of the equalities which this condition may imply. Given our assumptions that the maximand is jointly concave (B1) and that storage and retrieval require energy (B3), the KuhnTucker conditions uniquely define the global optimum associated with each specified level of terminal storage $(\hat{S}>0)$. For a formal proof, see (Salant et al., 1993, working paper).

\section{- The Four Possible Types of Optimal Behavior}

At the outset of our analysis, we verify formally that it is never optimal both to store new food and to retrieve from storage in the same period. Suppose to 
the contrary $R_{t}>0$ and $N_{t}>0$. Then Conditions 2 and 3 imply

$$
\text { and } \begin{aligned}
U^{\prime}\left(H_{t}+R_{t}-N_{t}\right)-\lambda_{t} \theta-\gamma_{t} \alpha_{r} & =0 \\
-U^{\prime}\left(H_{t}+R_{t}-N_{t}\right)+\lambda_{t} \theta-\gamma_{t} \alpha_{n} & =0 .
\end{aligned}
$$

Summing, we get

$$
-\gamma_{t}\left(\alpha_{n}+\alpha_{r}\right)=0
$$

But by (B3), the second factor is not zero; moreover, since (B1), (B3) and Condition 1 imply that the first factor $\left(-\gamma_{t}\right)$ is nonzero, Equation 11 cannot hold. Hence, in an optimal program either $R_{t}$ or $N_{t}$ (or both) must be zero. Intuitively, if the animal both stored and retrieved at the same time, it could reduce both activities marginally by the same amount, use the energy saved to forage for more food, and consume all of it. Even if the animal changed her behavior in no other way, her reproductive success would increase because the proposed perturbation increases her body weight without reducing the stockpile available when the litter arrives. Hence, programs where new storage and retrieval occur simultaneously never maximize fitness.

Next, we establish that it is always optimal to engage in some food-related activity $\left(A_{t}>0\right)$. Suppose to the contrary that $A_{t}=0$ for some $t$. As a result, $H_{t}=0, R_{t}=0$ and $C_{t}=0$. Then Conditions 1 and 10 reduce to:

$$
\begin{aligned}
& \frac{U^{\prime}(0)}{\alpha_{h(t)}} \leq \gamma_{t} \\
& F^{\prime}(0) \geq \gamma_{t} .
\end{aligned}
$$

But these inequalities are jointly inconsistent with B2. Therefore, it is optimal to expend some energy on food-related activities $\left(A_{t}>0\right)$ in every period. Simplifying Condition 10, we obtain:

$$
\text { 10'. } A_{t}>0 \text { and } F^{\prime}\left(A_{t}\right)=\gamma_{t} \text {. }
$$

The role of $\mathrm{B2}$ is to simplify the analysis by eliminating from consideration cases where $A_{t}=0$.

Since the other three behavioral variables $\left(H_{t}, R_{t}\right.$ and $\left.N_{t}\right)$ must each be either zero or strictly positive there are in principle $2^{3}$ cases to consider. But two of these eight cases involve $R_{t}>0$ and $N_{t}>0$ simultaneously and, as proved above, this cannot be optimal. A third case has $H_{t}=0, R_{t}=0$ but $N_{t}>0$. Since $C_{t}=H_{t}+R_{t}-N_{t}$, such a program would violate the constraint that consumption be nonnegative. A fourth case has $H_{t}=N_{t}=R_{t}=0$, which cannot be optimal since it implies that $A_{t}=0$.

\section{There remain 4 cases to consider:}

$\begin{array}{lccc} & H_{t} & R_{t} & N_{t} \\ \text { Case } 1 \text { (retrieval only) } & 0 & + & 0 \\ \text { Case } 2 \text { (harvesting and retrieval) } & + & + & 0 \\ \text { Case } 3 \text { (harvesting only) } & + & 0 & 0 \\ \text { Case } 4 \text { (harvesting and new storage) } & + & 0 & +\end{array}$

If fitness is maximized, behaviors must always fall within one of these four "regions" although transitions between them may occur as time passes.

\section{Pure Sieve Effects}

It turns out that if food is uniformly available before the onset of winter and uniformly unavailable thereafter (until the next litter arrives) as specified in B6, how each of the 5 behavioral variables changes in each of the four regions can, with a single exception, be predicted. That is, nineteen predictions summarized below in Table 1 are unambiguous while the twentieth, whether foraging $(H)$ is increasing or decreasing in region 4 , is ambiguous. If food availability varies in some region, then the predictions 
listed for that region in Table 1 would have to be modified as described more fully below for region 4

How each behavioral variable can be determined in each region is described in detail in the Appendix. For the sake of generality, the analysis there permits food availability to vary systematically over time. Each prediction in Table 1 is verified in the Appendix by specializing the analysis to the case of uniform availability of food before the onset of winter and uniform scarcity thereafter (B6). Assuming the animal has nothing in storage initially $(\bar{S}=0)$ and that the terminal stock $\hat{S}$ is not set so high as to preclude even the slightest retrieval, the Appendix also shows the order in which the regions will be encountered: initially the animal will forage without storing (region 3), then she will forage and store as winter approaches (region 4), then she will possibly cease foraging altogether and live off of her stockpile (region 1) before resuming foraging while continuing to retrieve (region 2). The Appendix presents a condition sufficient for region 1 to be skipped in this sequence.

\section{How Food Availability Effects and Sieve Effects Interact}

In the remainder of this section, we focus on the predictions in region 4 , which corresponds to hoarding behavior prior to the onset of winter. We first derive the pure sieve effect and then show how our predictions would be changed if food availability increased during the fall. While space considerations prevent us from considering in the text the other three cases, the interested reader is referred to the Appendix, which contains a parallel and complete treatment of them.

Throughout region 4, $H_{t}>0, N_{t}>0, A_{t}>0$ and $R_{t}=0$. Therefore, the Kubn-Tucker conditions imply that the following equations hold in region 4:

$$
\begin{gathered}
U^{\prime}\left(H_{t}-N_{t}\right)-\gamma_{t} \alpha_{h(t)}=0 \\
-U^{\prime}\left(H_{t}-N_{t}\right)+\lambda_{t} \theta-\alpha_{n} \gamma_{t}=0 \\
A_{t}-\alpha_{h(t)} H_{t}-\alpha_{n} N_{t}=0 \\
F^{\prime}\left(A_{t}\right)=\gamma_{t} .
\end{gathered}
$$

Adding Equations 1 and 3, we obtain:

$$
\gamma_{t}=\frac{\lambda_{t} \theta}{\alpha_{h(t)}+\alpha_{n}} .
$$

Inverting the function defined in Equation $10^{\prime}$ and substituting, we obtain:

$$
A_{t}=F^{\prime-1}\left(\gamma_{t}\right)
$$

Noting that $C_{t}=H_{t}-N_{t}$ in region 4 , and inverting the function defined in Equation 1 we obtain:

$$
C_{t}=U^{\prime-1}\left(\alpha_{h(t)} \gamma_{t}\right)
$$

Finally, using Equation 8 we conclude:

$$
H_{t}=\frac{A_{t}+\alpha_{n} C_{t}}{\alpha_{h(t)}+\alpha_{n}}
$$

and

$$
N_{\mathrm{t}}=\frac{A_{\mathrm{t}}-\alpha_{h(\ell)} C_{\mathrm{t}}}{\alpha_{h(\mathrm{t})}+\alpha_{\mathrm{n}}}
$$

Equations 12-16 define five variables- $\gamma_{t}, A_{t}, C_{t}, H_{t}$ and $N_{t}$ as continuous functions of $\lambda_{t}$ and $\alpha_{h(t)}$ in region 4. Since storage is positive throughout this region, Condition 4 implies that $\left\{\lambda_{t}\right\}$ is strictly increasing when the stockpile is leaky $(\theta<1)$ and constant in the extreme case where all storage can be retrieved.

It is instructive first to assume that (a) all storage is retrievable $(\theta=1)$ and (b) food is uniformly available before the onset of winter (B6). Then, since both $\left\{\lambda_{t}\right\}$ and $\left\{\alpha_{h(t)}\right\}$ are constant sequences, the five equations indicate that $\left\{\gamma_{t}\right\},\left\{A_{t}\right\},\left\{C_{t}\right\},\left\{N_{t}\right\}$ and $\left\{H_{\imath}\right\}$ are constant sequences throughout region 4. This constancy is reminiscent of the equal replacement-time result in our first example.

Next we maintain the assumption that food is uniformly available but drop the assumption of a perfect stockpile. This permits us to isolate pure sieve effects. Since Condition 4 implies that $\left\{\lambda_{t}\right\}$ strictly increases, Equation 12 implies that $\left\{\gamma_{t}\right\}$ strictly 
increases. But since the function $F^{\prime-1}(\cdot)$ is strictly increasing and the function $U^{\prime-1}(\cdot)$ is strictly decreasing (B1), Equations 13 and 14 in turn imply that $\left\{A_{t}\right\}$ strictly increases and $\left\{C_{t}\right\}$ strictly decreases. $\left\{N_{t}\right\}$ must strictly increase since, as Equation 16 reflects, it is the difference between an increasing and a decreasing sequence. Nothing can be said about the foraging sequence $\left\{H_{\mathrm{t}}\right\}$ since, as Equation 15 reflects, it is the sum of a decreasing and an increasing sequence. These pure sieve effects are what we recorded in Table 1. The predictions in Table 1 for the other three regions reflect conclusions derived in the same manner in the Appendix.

Finally, we show how the analysis can be modified in the case where food becomes more available before the onset of winter. Changes in the availability of food reinforce some sieve effects but offset others. For example, if $\left\{\alpha_{h(t)}\right\}$ is a decreasing sequence a re-analysis of Equations 12 and 13 confirms that both $\left\{\gamma_{t}\right\}$ and $\left\{A_{t}\right\}$ increase over time as before since $\left\{\lambda_{t}\right\}$ strictly increases throughout this region. On the other hand, the sieve effect and food-availability effect have conflicting influences on consumption. To see this, note that from Equation 12:

$$
\alpha_{h(t)} \gamma_{t}=\frac{\lambda_{t} \theta}{1+\frac{a_{n}}{\alpha_{h(t)}}}
$$

Hence

$$
\frac{\alpha_{h(t)} \gamma_{t}}{\alpha_{h(t+1)} \gamma_{t+1}}=\frac{\theta\left(1+\frac{\alpha_{a}}{\alpha_{h(t+1)}}\right)}{\left(1+\frac{\alpha_{l}}{\alpha_{M(t)}}\right)} .
$$

Since $C_{t}$ is a strictly decreasing function of the product, $\alpha_{h(t)} \gamma_{t}$, Equations 1 and 18 imply:

$$
C_{t} \geq C_{t+1} \Leftrightarrow 1+\frac{\alpha_{n}}{\alpha_{h(t)}} \geq \theta\left(1+\frac{\alpha_{n}}{\alpha_{h(t+1)}}\right) .
$$

These inequalities imply that, if storage were perfect, consumption would increase with food availability. If, on the other hand, food were uniformly available, consumption would decrease due to the sieve effect. Hence, if food becomes more available over time in region 4 , the two effects would work in opposite directions. Whether consumption strictly increases or not depends on the magnitudes of four exogenous variables: $\alpha_{h(t)}, \alpha_{h(t+1)}, \alpha_{n}$, and $\theta$.

If the sieve effect dominates, then $\left\{C_{t}\right\}$ will decrease as before. In addition, since the numerator on the right of Equation 16 will increase over time while the denominator will decrease, $\left\{N_{t}\right\}$ will increase as before.

If, on the other hand, the food-availability effect dominates, then $\left\{C_{t}\right\}$ will increase over time. In this case, the previous ambiguity about foraging disappears: $\left\{H_{t}\right\}$ strictly increases since the numerator on the right of Equation 15 increases over time while the denominator decreases. In this circumstance, however, the direction of change of $\left\{N_{t}\right\}$ becomes ambiguous.

The reader can easily modify the pure sieve effects in the other three rows of Table 1 to account for variations in food availability by analyzing the equations in the Appendix which correspond to each of the other regions.

\section{DISCUSSION}

In this paper, we explained a methodology which permits the deduction of many qualitative implications of the hypothesis of fitness maximization without a detailed knowledge of the form of the fitness function. As a first step, we identified an optimization subproblem which must be solved if fitness is maximized but which does not itself involve the fitness function. As a second step, we showed that solutions to this subproblem must have certain qualitative characteristics; these characteristics are, therefore, implications of fitness maximization. In addition, we showed how sometimes elaborate quantitative predictions can be made from the analysis of this subproblem when a limited amount of additional information can be observed.

We developed these ideas, which are fundamental to economics, in terms of two biological examples. These examples were deliberately simplified to permit the clearest exposition of a novel methodology. Thus, our assumptions about the weight gain function $\left(G\left(C_{t}, A_{t}, W_{t}, t\right)\right)$ were intended to facilitate analysis rather than to reflect the details of what is now known about the effects of food intake and activity on the 
weight of mammals in different climates. Similarly, we excluded from consideration an aspect of hoarding behavior which is unquestionably important: animals can adopt any of a wide variety of different hoarding techniques for preparing, transporting, placing, and concealing stored food as catalogued in Vander Wall (1991).

The validity of our methodology in no way depends on these simplifications. Now that the methodology has been clarified, it is both feasible and appropriate to add greater detail and realism to our two examples. For instance, to predict which of a finite number of hoarding strategies an animal would adopt, each alternative strategy mentioned by Vander Wall could be characterized by its three stockpiling parameters $\left(\theta, \alpha_{n}\right.$, and $\left.\alpha_{r}\right)$. The choice of hoarding strategy (regarded as a discretized rather than a continuous decision variable) could be predicted in the same manner as the other behavioral variables in the model. Geometrically, there would be a different "transformation curven like that in Figure 2 associated with each hoarding strategy. Fitness maximization would then require using whatever transformation curve had the highest vertical component for each given horizontal component. That is, the analog of Figures 1 and 2 could be constructed from the outer envelope of this finite set of transformation curves.

In a paper about tradeoffs and fitness, it is of course fitting that we note the fundamental tradeoff in modelling: the more realistic and detailed the assumptions, the more complicated and unwieldy the resulting model. If, however, quantitative predictions alone are desired, even relatively complicated variations on our hoarding example can be incorporated into simple spreadsheet simulations. We find such spreadsheet simulations are also useful in suggesting qualitative properties of such models which can then be investigated analytically. A spreadsheet of our hoarding example is available upon request.

To reiterate, we simplified our two examples so that we could explain an unfamiliar methodology in the clearest fashion. It would be entirely mistaken for readers to conclude from these simplifications that our methodology only applies to models which lack biological realism.

We also chose to analyze the optimization problem in each example using calculus rather than dynamic programming (Mangel and Clark 1988, p.233). Either technique could have been used: our paper concerns the structure of optimization problems, not the mere mathematical technique by which they are solved. We chose calculus because it seemed better suited to the derivation of analytical results. When the research focus changes to model estimation and hypothesis testing, however, dynamic programming may well be the more appropriate technique. 


\section{APPENDIX:}

Characterization of the Optimal Time Paths Before and After the Onset of Winter

In this Appendix, we determine how each behavioral variable changes within each of the four regions by analyzing the first-order conditions given in Conditions 1-10 of the text. Numbers below refer to those conditions. We verify the sieve effects reported in Table 1 for each region for the special case where B6 holds. Readers wishing to explore cases where food availability varies in arbitrary ways for regions 1,2 ,or 3 may want first to review the analysis in the text of how pure sieve effects and food availability effects interact in region 4.

In addition, this Appendix indicates the order in which the regions will be reached in the case where food is uniformly available before winter and uniformly scarce thereafter. Finally, we present a condition on the exogenous parameters sufficient for region 1 to be skipped in any optimal program.

We show below that $A, C, H, N$, and $R$ can be written as a continuous function of the multiplier $\lambda$. There are two sets of these five functions-one set holds prior to the onset of winter and the other set holds subsequent to its onset.

Prior to the onset of winter, optimal behavior on a given period depends on $\lambda$ in that period, $\lambda$ must lie in one of the disjoint regions which correspond to the cases above. If $\lambda$ is in region $1,2,3$, or 4 then case $1,2,3$ or 4 (respectively) arises. The boundary between region $i$ and $j$ at date $t$ is denoted $\lambda_{i, j}^{t}$.

Below, we show how the variables $A_{t}, H_{t}, R_{t}, N_{t}$, and $C_{t}$ can be deduced as functions of $\lambda_{t}$ for $\lambda_{t}$ in any of four possible regions.

Region 1 (retrieval only): In region $1, N_{t}=H_{t}=0, A_{t}>0$ and $R_{t}>0$. The following equations must hold (the numbers refer to the corresponding Kuhn-Tucker conditions);

$$
\begin{gathered}
U^{\prime}\left(R_{t}\right)-\lambda_{t} \theta-\gamma_{t} \alpha_{r}=0 \\
A_{t}-\alpha_{r} R_{t}=0
\end{gathered}
$$

$$
F^{\prime}\left(A_{t}\right)=\gamma_{t}
$$

Combining Equations 2, 8, and 10 we obtain:

$$
U^{\prime}\left(R_{\imath}\right)-\lambda_{t} \theta-F^{\prime}\left(\alpha_{r} R_{t}\right) \alpha_{r}=0 .
$$

This uniquely defines $R_{t}$ (implicitly) as a strictly decreasing function of $\lambda_{t}$.

In addition, Equation 8 implies:

$$
A_{t}=\alpha_{r} R_{t}
$$

Hence $A_{t}$ is a strictly increasing function of $R_{t}$ and, therefore, a strictly decreasing function of $\lambda_{t}$. Since $N_{t}=H_{t}=0, C_{t}=R_{t}$. Hence every behavioral variable depends in region 1 on $\lambda_{t}$. As $\lambda_{t}$ increases in region $1, R_{t}, A_{t}$, and $C_{t}$ strictly decrease.

Region 2 (harvesting and retrieval):

In region $2, N_{t}=0, H_{t}>0, A_{t}>0$, and $R_{t}>0$. The following equations must hold:

$$
\begin{gathered}
U^{\prime}\left(H_{t}+R_{t}\right)-\gamma_{t} \alpha_{h(t)}=0 \\
U^{\prime}\left(H_{t}+R_{t}\right)-\lambda_{t} \theta-\gamma_{t} \alpha_{t}=0 \\
A_{t}-\alpha_{h(t)} H_{t}-\alpha_{r} R_{t}=0 \\
F^{\prime}\left(A_{t}\right)=\gamma_{t} .
\end{gathered}
$$

After subtracting Equation 2 from Equation 1 and simplifying, we obtain:

$$
\gamma_{t}=\frac{\lambda_{t} \theta}{\alpha_{h(t)}-\alpha_{r}}
$$

Inverting the function defined in Equation 10, we find:

$$
A_{t}=F^{\prime-1}\left(\gamma_{t}\right)=F^{\prime-1}\left(\frac{\lambda_{t} \theta}{\alpha_{h(t)}-\alpha_{r}}\right)
$$

Hence $A_{t}$ is a strictly increasing function of $\lambda_{t}$ in region 2. Since $C_{t}=H_{t}+R_{t}$, Equation 1 implies

$$
C_{t}=U^{\prime-1}\left(\gamma_{t} \alpha_{h(t)}\right)=U^{\prime-1}\left(\frac{\lambda_{t} \theta \alpha_{h(t)}}{\alpha_{h(t)}-\alpha_{r}}\right)
$$


Hence in region 2, $C_{t}$ is a strictly decreasing function of $\lambda_{t}$.

Finally, from Equation 8, we obtain:

$$
\begin{gathered}
H_{t}=\frac{A_{t}-\alpha_{r} C_{t}}{\alpha_{h(t)}-\alpha_{r}} \\
R_{t}=\frac{\alpha_{h(t)} C_{t}-A_{t}}{\alpha_{h(t)}-\alpha_{r}} .
\end{gathered}
$$

Since $A_{t}$ and $C_{t}$ are uniquely determined by $\lambda_{t}$ so are $H_{t}$ and $R_{t}$. In region 2 , as $\lambda_{t}$ increases over time $H_{t}$ strictly increases while $R_{t}$ strictly decreases.

\section{Region 3 (harvesting only):}

In region $3, R_{t}=N_{t}=0, A_{t}>0$, and $H_{t}>0$. The following equations must hold:

$$
\begin{gathered}
U^{\prime}\left(H_{t}\right)-\gamma_{t} \alpha_{h(t)}=0 \\
A_{t}-\alpha_{h(t)} H_{t}=0 \\
F^{\prime}\left(A_{\imath}\right)=\gamma_{\imath} .
\end{gathered}
$$

Combining Equations 1, 8, and 10 we conclude:

$$
U^{\prime}\left(\frac{A_{t}}{\alpha_{h(t)}}\right)-F^{\prime}\left(A_{t}\right) \alpha_{h(t)}=0 .
$$

Hence $A_{t}$ in region 3 is independent of $\lambda_{t}$ and is constant if $\alpha_{h(t)}$ is constant. From Equation 8

$$
H_{t}=\frac{A_{t}}{\alpha_{h(t)}} .
$$

Moreover, since $C_{t}=H_{t}+R_{t}-N_{t}, C_{t}=H_{t}$. Note that in region $3, N_{t}=R_{t}=0$ and $A_{t}, H_{t}, C_{t}$ remain constant provided $\alpha_{h(t)}$ is constant.

\section{Region 4 (harvesting and new storage)}

Throughout region 4, $H_{t}>0, N_{t}>0, A_{t}>0$ and $R_{t}=0$. The following equations hold in region 4:

$$
U^{\prime}\left(H_{t}-N_{t}\right)-\gamma_{t} \alpha_{h(t)}=0
$$

$$
\begin{gathered}
-U^{\prime}\left(H_{t}-N_{t}\right)+\lambda_{t} \theta-\alpha_{n} \gamma_{t}=0 \\
A_{t}-\alpha_{h(t)} H_{t}-\alpha_{n} N_{t}=0 \\
F^{\prime}\left(A_{t}\right)=\gamma_{t} .
\end{gathered}
$$

Adding Equations 1 and 3, we obtain:

$$
\gamma_{t}=\frac{\lambda_{t} \theta}{\alpha_{h(t)}+\alpha_{n}} .
$$

Hence $\gamma_{t}$ is a strictly increasing function of $\lambda_{t}$. Inverting the function defined in Equation 10 and substituting, we obtain:

$$
A_{\mathrm{t}}=F^{-1}\left(\gamma_{\mathrm{t}}\right)
$$

Since $A_{t}$ is a strictly increasing function of $\gamma_{t}, A_{t}$ is a strictly increasing function of $\lambda_{t}$ in region 4. Noting that $C_{t}=H_{t}-N_{t}$ in region 4 , and inverting the function defined in Equation 1 we obtain:

$$
C_{t}=U^{\prime-1}\left(\alpha_{h(t)} \gamma_{t}\right)
$$

Hence $C_{t}$ is a strictly decreasing function of $\gamma_{t}$ and thus a strictly decreasing function of $\lambda_{t}$ in region 4. Finally using Equation 8 we conclude:

$$
H_{t}=\frac{A_{t}+\alpha_{n} C_{t}}{\alpha_{h(t)}+\alpha_{n}}
$$

and

$$
N_{t}=\frac{A_{t}-\alpha_{h(t)} C_{t}}{\alpha_{h(t)}+\alpha_{n}}
$$

Hence $N_{t}$ is a strictly increasing function of $\lambda_{t}$ in region 4. $H_{t}$ is the sum of a decreasing function and an increasing function of $\lambda_{t}$. As $\lambda_{t}$ increases in region 4, $A_{t}$ and $N_{t}$ strictly increase, $C_{t}$ strictly decreases, and $H_{t}$ may either increase or decrease.

\section{Defining the Boundaries of Each Region}

In region 1, $H_{t}=0$; in region 2, $H_{t}>0$ and strictly increases. Equations 2, 8, and 10 hold in both regions, but Equation 1 which must hold with equality when $H_{t}>0$, 
need not hold in region 1. To determine the boundary between region 1 and 2, we find that value for $\lambda_{t}$, denoted $\lambda_{1,2}$, such that $H_{t}=0$ in region 2 . Since

$$
\begin{gathered}
H_{t}=\frac{A_{t}-\alpha_{r} C_{t}}{\alpha_{h(t)}-\alpha_{r}} \\
H_{t}=0 \Rightarrow \alpha_{r} C_{t}=A_{t} \\
\text { or } \alpha_{r} U^{\prime-1}\left(\frac{\lambda_{1,2} \theta \alpha_{h(t)}}{\alpha_{h(t)}-\alpha_{r}}\right)=F^{\prime-1}\left(\frac{\lambda_{1,2} \theta}{\alpha_{h(t)}-\alpha_{r}}\right) .
\end{gathered}
$$

This equation uniquely defines $\lambda_{1,2}$. The boundary, $\lambda_{1,2}$, changes over time only if $\alpha_{h(t)}$ changes.

In region $2, R_{t}>0$ and strictly decreases; in region $3, R_{t}=0$. Equations 1,8 , and 10 hold in both regions but Equation 2, which must hold with equality when $R_{t}>0$, need not hold in region 3 . To determine the boundary between regions 2 and 3 , we find that value for $\lambda_{t}$, denoted $\lambda_{2,3}$, such that $R_{t}=0$ in region 2 . Since in region 2

$$
\begin{gathered}
R_{t}=\frac{\alpha_{h(t)} C_{t}-A_{t}}{\alpha_{h(t)}-\alpha_{r}} \\
R_{t}=0 \Rightarrow \alpha_{h(t)} C_{t}-A_{t}=0 \\
\text { or } \alpha_{h(t)} U^{\prime-1}\left(\frac{\lambda_{2,3} \theta \alpha_{h(t)}}{\alpha_{h(t)}-\alpha_{r}}\right)=F^{\prime-1}\left(\frac{\lambda_{2,3} \theta}{\alpha_{h(t)}-\alpha_{r}}\right) .
\end{gathered}
$$

This equation uniquely determines $\lambda_{2,3}$. The boundary, $\lambda_{2,3}$, changes over time only if $\alpha_{h(t)}$ changes.

In region $3, N_{t}=0$; in region $4, N_{t}>0$. Equations 1,8 , and 10 hold in both regions but Equation 3, which must hold with equality when $N_{t}>0$ need not hold in region 3 . To determine the boundary between regions 3 and 4 , we find that value for $\lambda_{t}$, denoted $\lambda_{3,4}$, such that $N_{t}=0$ in region 4 . Since in region 4

$$
\begin{gathered}
N_{t}=\frac{A_{t}-\alpha_{h(t)} C_{t}}{\alpha_{h(t)}+\alpha_{n}} \\
N_{t}=0 \Rightarrow A_{t}=\alpha_{h(t)} C_{t} \\
\text { or } F^{\prime-1}\left(\frac{\lambda_{3,4} \theta}{\alpha_{h(t)}+\alpha_{n}}\right)=\alpha_{h(t)} U^{\prime-1}\left(\frac{\alpha_{h(t)} \lambda_{3,4} \theta}{\alpha_{h(t)}+\alpha_{n}}\right) .
\end{gathered}
$$

This equation uniquely determines $\lambda_{3,4}$. The boundary, $\lambda_{3,4}$, changes over time only if $\alpha_{h(t)}$ changes.

\section{How the Regions Fit Together}

Next we verify that

$$
\lambda_{1,2}<\lambda_{2,3}<\lambda_{3,4}
$$

Reconsider the equation defining $\lambda_{1,2}$. Geometrically it can be regarded as the intersection of a downward-sloping function of $\lambda$ and an upward-sloping function of

Reconsider the equation defining $\lambda_{2,3}$. Notice that it too can be regarded as defined by the intersection of an upward and a downward-sloping functions of $\lambda$. Indeed, the upward-sloping function in each case is identical:

$$
F^{\prime-1}\left(\frac{\lambda \theta}{\alpha_{h(t)}-\alpha_{r}}\right)
$$

However, the downward-sloping function defining $\lambda_{2,3}$ lies uniformly above the downwardsloping function defining $\lambda_{1,2}$. That is:

$$
\alpha_{h(t)} U^{\prime-1}\left(\frac{\lambda \theta \alpha_{h(t)}}{\alpha_{h(t)}-\alpha_{r}}\right)>\alpha_{r} U^{\prime-1}\left(\frac{\lambda \theta \alpha_{h(t)}}{\alpha_{h(t)}-\alpha_{r}}\right)
$$

since $\alpha_{h(t)}>\alpha_{r}$ by B4. This implies that $\lambda_{2,3}>\lambda_{1,2}$.

Next, reconsider the equation defining $\lambda_{3,4}$. It can be regarded as the intersection of an upward-sloping function of $\lambda$ and a downward-sloping function of $\lambda$. Note that, given B3, the upward-sloping function defining $\lambda_{3,4}$ is uniformly smaller than the upward-sloping function defining $\lambda_{2,3}$ :

$$
F^{\prime-1}\left(\frac{\lambda \theta}{\alpha_{h(t)}+\alpha_{n}}\right)<F^{\prime-1}\left(\frac{\lambda \theta}{\alpha_{h(t)}-\alpha_{r}}\right) .
$$

B3 also implies that the downward-sloping function defining $\lambda_{3,4}$ is uniformly larger than the downward-sloping function defining $\lambda_{2,3}$

$$
U^{\prime-1}\left(\frac{\alpha_{h(t)} \lambda \theta}{\alpha_{h(t)}+\alpha_{n}}\right)>U^{\prime-1}\left(\frac{\alpha_{h(t)} \lambda \theta}{\alpha_{h(t)}-\alpha_{r}}\right) .
$$

This implies that $\lambda_{3,4}>\lambda_{2,3}$

Figure 3 illustrates how the intervals fit together:

These boundaries are ordered in this way regardless of the value of $\alpha_{h(t)}$. 
For simplicity, consider the case where $\alpha_{h(t)}$ is constant until the onset of winter and then jumps up to a new constant level. Consider the equations defining $\lambda_{1,2}$. When $\alpha_{h(t)}$ jumps up, the upward-sloping function shifts down while the downwardsloping function shifts up. Consequently, the boundary, $\lambda_{1,2}$ jumps up. For the same reason, the boundary $\lambda_{2,3}$ must jump up. As a result $\lambda_{t}$ can increase by $1 / \theta$ at the onset of winter and nonetheless transit from region 4 to region 1 or 2 as discussed in the text.

\section{A Condition Sufficient for No Hibernation}

To conclude this appendix, we present a condition sufficient for region 1 not to occur in an optimal program.

If $\alpha_{h(t+1)}<\frac{a_{M(1)}}{l}+\left(\alpha_{r}+\frac{a_{n}}{0}\right)$, the transition will be to region 2. By hypothesis

$$
\theta<\frac{\alpha_{h(\imath)}+\alpha_{n}}{\alpha_{n(\imath+1)}-\alpha_{r}}
$$

Hence

$$
\theta=\frac{\lambda_{t}}{\lambda_{t+1}}<\frac{\alpha_{h(t)}+\alpha_{n}}{\alpha_{h(t+1)}-\alpha_{r}}
$$

Therefore,

$$
\alpha_{h(t)} U^{\prime-1}\left(\frac{\lambda_{t} \theta \alpha_{h(t)}}{\alpha_{h(t)}+\alpha_{n}}\right)>\alpha_{r} U^{\prime-1}\left(\frac{\lambda_{t+1} \theta \alpha_{h(t+1)}}{\alpha_{h(t+1)}-\alpha_{r}}\right) .
$$

Recall that since $\lambda_{t}>\lambda_{3,4}$

$$
\alpha_{h(t)} U^{\prime-1}\left(\frac{\alpha_{h(t)} \lambda_{t} \theta}{\alpha_{h(t)}+\alpha_{n}}\right) \leq F^{\prime-1}\left(\frac{\lambda_{t} \theta}{\alpha_{h(t)}+\alpha_{n}}\right) .
$$

Hence

$$
\alpha_{r} U^{\prime-1}\left(\frac{\lambda_{t+1} \theta \alpha_{h(t+1)}}{\alpha_{h(t+1)}-\alpha_{r}}\right) \leq F^{\nu-1}\left(\frac{\lambda_{t} \theta}{\alpha_{h(t)}+\alpha_{n}}\right) .
$$

But the boundary $\lambda_{1,2}^{i+1}$ implicitly solves

$$
\alpha_{r} U^{\prime-1}\left(\frac{\lambda_{1,2}^{t+1} \theta \alpha_{h(t+1)}}{\alpha_{h(t+1)}-\alpha_{r}}\right)=F^{\prime-1}\left(\frac{\lambda_{1,2}^{t+1} \theta}{\alpha_{h(t+1)}-\alpha_{r}}\right) .
$$

Suppose $\lambda^{t+1} \leq \lambda_{1,2}^{t+1}$. Then

$$
\alpha_{r} U^{\prime-1}\left(\frac{\lambda_{t+1} \theta \alpha_{h(t+1)}}{\alpha_{h(t+1)}-\alpha_{r}}\right) \geq F^{\prime-1}\left(\frac{\lambda_{t}}{\alpha_{h(t+1)}-\alpha_{r}}\right)
$$

$$
F^{\prime-1}\left(\frac{\lambda_{t} \theta}{\alpha_{h(t)}+\alpha_{n}}\right) \geq F^{\prime-1}\left(\frac{\lambda_{t}}{\alpha_{h(t+1)}-\alpha_{r}}\right) .
$$

But since $F^{\prime-1}(\cdot)$ is increasing and $\theta<\frac{a_{M(1)}+\alpha_{n}}{a_{M(1+1)}-a_{r}}$, this inequality cannot hold. Therefore, $\lambda^{t+1}>\lambda_{1,2}^{t+1}$.

A necessary condition for a transition to region 1 is therefore that $\alpha_{h(t+1)} \geq$ $\frac{a_{n}(t)+a_{n}}{\theta}+\alpha_{r}$. 


\section{References}

Baumol WJ, 1977.Economic Theory and Operations Analysis. Englewood Cliffs, New Jersey: Prentice Hall.

Dorfman R, Solow R, Samuelson P, 1987.Linear Programming and Economic Analysis. New York, New York: Dover Publications.

Layard PRG, Walters AA, 1987.Microeconomic Theory. New York, New York : McGraw Hill.

Mangel M, Clark CW, 1988.Dynamic Modeling in Behavioral Ecology. Princeton, New Jersey: Princeton University Press.

Schoen DJ, Ashman T-L, "The evolution of floral longevity: Resource allocation to maintenance versus construction of repeated structures in modular organisms," Evolution, , in press.

Stephens D, Krebs JR, 1986.Foraging Theory. Princeton, New Jersey: Princeton University Press.

Vander Wall S, 1991.Food Hoarding in Animals. Chicago, Illinois: University of Chicago Press.

\section{Acknowledgments}

We would like to thank Tim Livingston and Raquel Noriega for their early contributions to this research. We would like to thank Dan Schoen, whose research on flower replacement in annuals led to our preliminary example on perennials. We would also like to thank the following readers whose advice, sometimes on multiple drafts. we found particularly useful: Carl Bergstrom, Gardner Brown, Colin Clark, Jon Fausl. Miles Kimball. Donald Kramer. Bobbi Low, Jeff Lucas, Marc Mangel, Gordon Orians, Alan Rogers, David Simpson, Ted Sims, David Stephens, Stephen Vander Wall, and two anonymous referees of this journal. Finally, we would like to express appreciation to Florence Chauvet for her efficient typing. Salant's research was supported by Resources for the Future, where he was a Gilbert White Fellow (1992-3) and by the Institut d'Économie Industrielle and the Institut. National de la Recherche Agronomique in Toulousc. Prance where he was a visitor in June. 1994. 


\section{FIGURE LEGENDS}

Figure 1-Given the initial energy reserves of a perennial, the number of flowers it forms during the season and the length of time each is maintained determines the number of seeds produced and the energy remaining at the season's end. Each point in the diagram represents one resulting combination of seeds produced and energy remaining. Combinations on the downward-sloping boundary of Figure 1 or between that boundary and the axes can be achieved while combinations outside the downward-sloping boundary are infeasible. Provided fitness is a strictly increasing function of one of the currencies on the axes, fitness maximization must result in an outcome somewhere on the downward-sloping boundary. The boundary can be achieved only if each flower is maintained for an equal length of time. Determining that length and the number of flowers which will form requires more information. If the end-of-season energy reserves were observed to be $\hat{E}^{*}$, then the number of flowers produced and the common time each is replaced could be predicted as the determinants of the boundary point directly above $\hat{E}^{\bullet}$.

Figure 2-Given her initial body weight, the foraging and hoarding behavior of a female mammal between the prior summer and the arrival of her first post-winter litter determines the size of her external stockpile as well as her own body weight following the birth of that litter. Each point in the diagram represents one resulting combination of body weight and stockpile size. As in Figure 1, combinations either on the downward-sloping boundary or between that boundary and the axes can be achieved while combinations outside the downward-sloping boundary are infeasible. If fitness is maximized, the resulting combination of body weight and stockpile size must lie somewhere on the downward-sloping boundary. Such combinations have distinctive characteristics (see Table 1). These qualitative implications of fitness maximization can be supplemented by quantitative predictions if, for example, the animal's body weight is measured following the birth of her litter.
Figure 3-The Kuhn-Tucker conditions (1-10) require that the multiplier $(\lambda)$ be nonnegative and that it grow between periods by the factor $\theta^{-1}(>1)$ once stockpiling begins. The diagram divides the nonnegative real line into four disjoint regions ( $R$ IR4) with $\lambda_{i, j}$ denoting the boundary between region $i$ and $j$. Increases in the energy cost of foraging $\left(\alpha_{n(t)}\right)$ cause the boundaries of the regions to shift up while decreases in the cost of foraging cause them to shift down; the boundaries do not change when this cost is constant. As Table 1 summarizes, the qualitative behavior of the animal in a particular period depends on which region contains $\lambda$ during that period. On the period before the onset of winter, $\lambda$ is contained in Region 4 . When winter arrives, the cost of foraging increases and the boundaries of the regions shift up; consequently $\lambda$-which continues to increase geometrically-may fall into Region 1 or 2 . 
Table 1

Pure Sieve Effects: How Behavioral Variables Change Within a Region as Time Elapses

Variable

Region

Region 1 (retrieval only)

$\begin{array}{lllllllllll}H & R & N & C & A\end{array}$

Region 2 (harvesting and retrieval)

$0 \downarrow 1 \quad 0 \quad \downarrow \quad 1$

Region 3 (harvesting only)

$\uparrow \quad 1 \quad 0 \quad \downarrow \quad \uparrow$

$\rightarrow 00 \rightarrow-$

Region 4 (harvesting and new storage) ? $\quad 0 \quad \uparrow \quad 1 \quad \uparrow$

Key: $\uparrow$ means a positive variable strictly increases over time. $\downarrow$ means a positive variable strictly decreases over time.

$\rightarrow$ means a positive variable is constant over time.

? means a positive variable could increase or decrease over time.

0 means a variable remains zero over time.

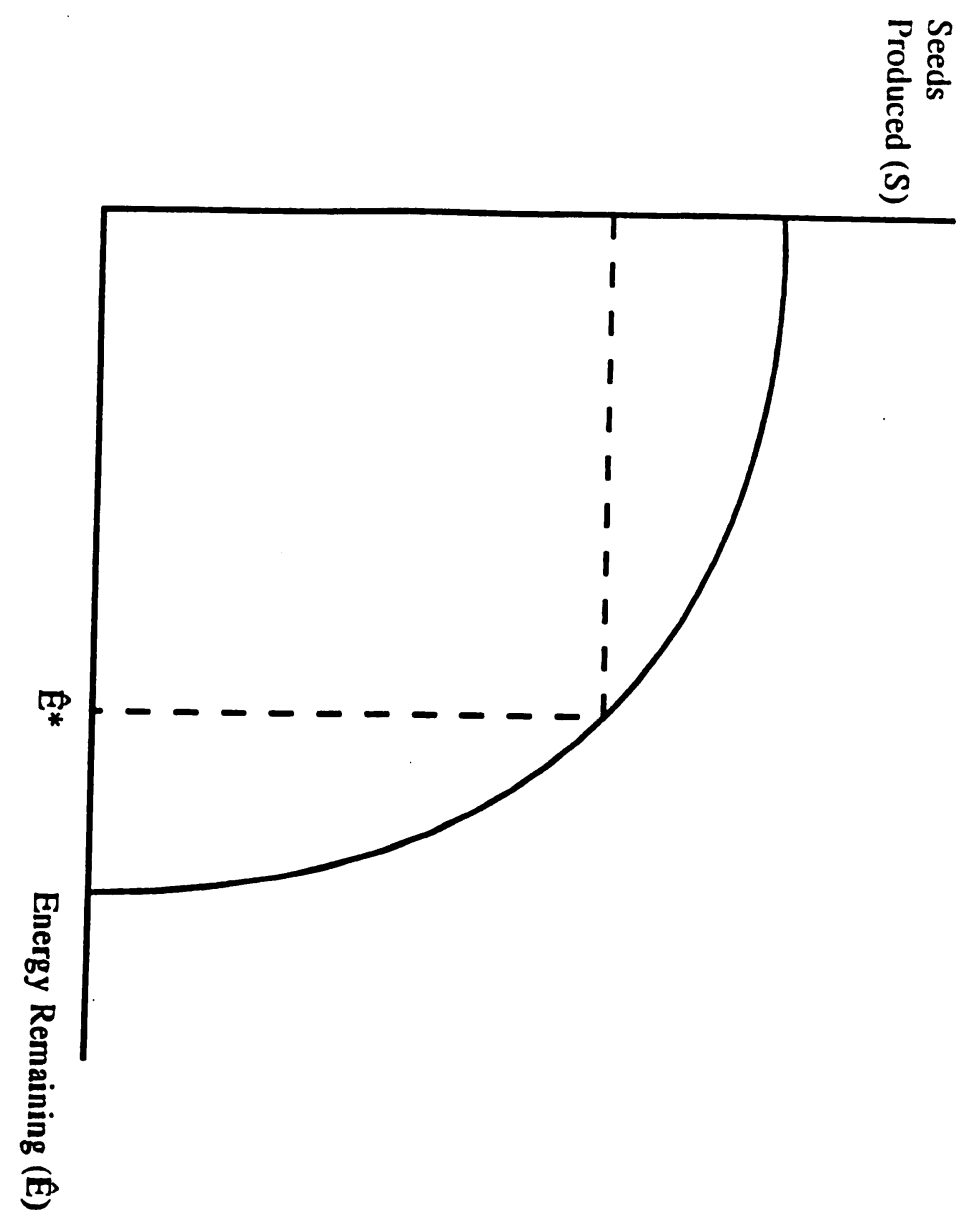




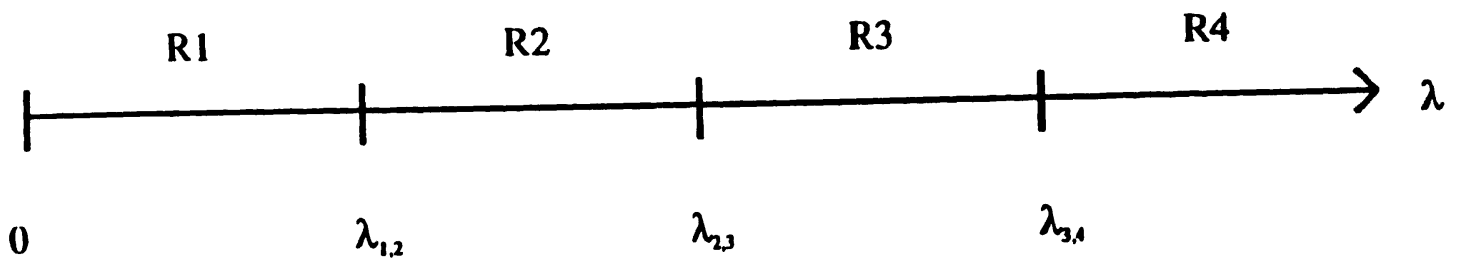

F.; 2 .

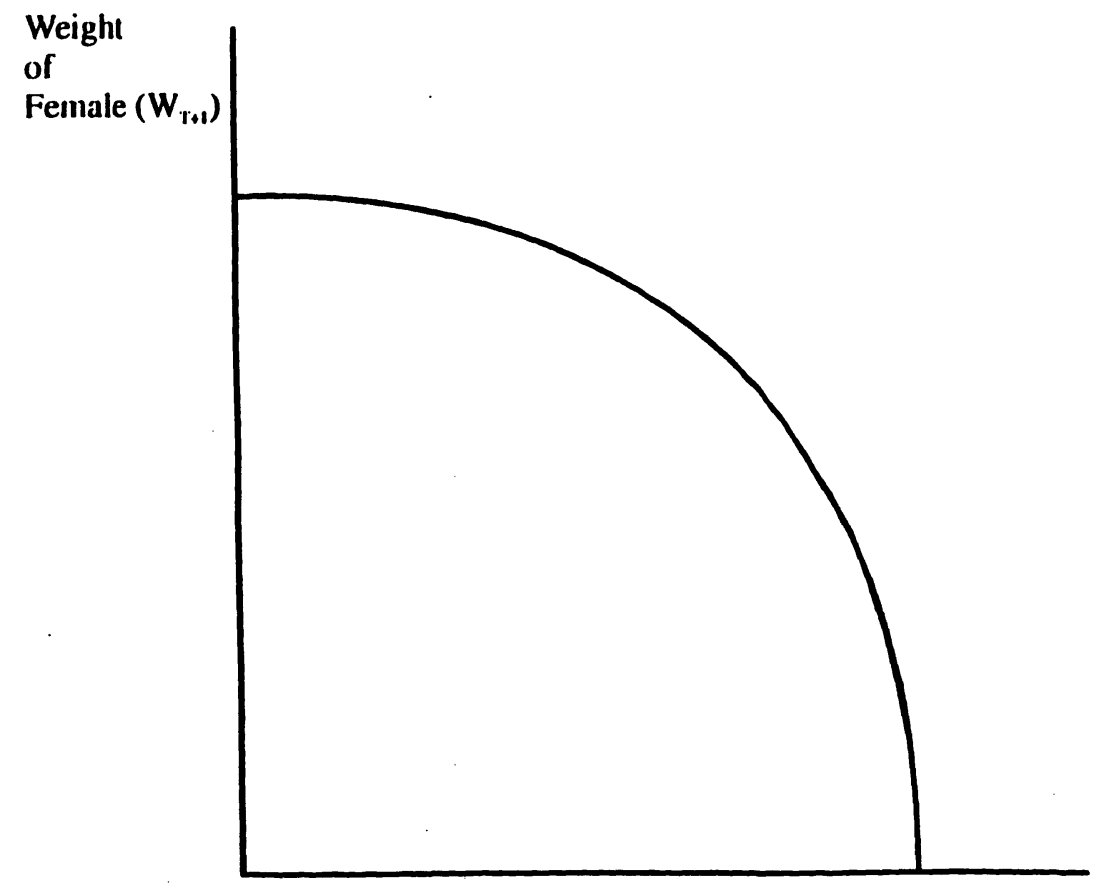

Stockpile Size $\left(S_{T+1}\right)$ 
DEMCO 

